2

\title{
Validation of a Novel Connected "Smart" Stoma Bag to Monitor Output in Ostomates
}

\author{
Robert I. Fearn ${ }^{1 *}$, Anupriya J. T. Naik ${ }^{2}$, Irina Dorofeeva ${ }^{2}$, Yumeng $\mathrm{Wu}^{2}$, David Ramirez ${ }^{2}$, Pamela \\ A. Borden ${ }^{2}$, Chris Landon ${ }^{3}$ \\ 1 Academic Department of Medical \& Surgical Gastroenterology, Homerton University Hospital, London, \\ United Kingdom; Robert.Fearn@nhs.net \\ 2 Department of Engineering, 11 Health and Technologies Inc., Tustin, California; Anupriya@11health.com \\ 3 Department of Pediatric Allergy and Immunology, Ventura County Medical Center, Ventura County, \\ California; Chris.Landon@ventura.org \\ * Correspondence: Robert.Fearn@nhs.net
}

\begin{abstract}
Over 55\% of stoma patients suffer complications such as dehydration. Outcomes may be improved through communicating stoma output data to the patient and their clinical teams. Past artificial neural networks to improve accuracy in fluid level sensing were designed to account for 'slosh' caused by variable acceleration in one or two axes of movement. This paper describes the development of a novel sensor platform for noninvasive monitoring of stoma output in real time through incorporating a volumetric array consisting of thermistors and capacitive sensors into an ostomy appliance. Stoma output which exits the body at core temperature passes into a stoma appliance in a pattern which is dictated by water content, existing effluent within the bag and distortion of the usual bag shape. By using thermistors, a thermal boundary demarcates the accumulated level of fecal material as the effluent settles. A capacitive array allows the measurement of volume of output. The sensing components communicates via near field communication (NFC) and transmits data to a smartphone application by Bluetooth low energy (BLE). Testing of the device on 11 existing ileostomy patients with 51.6 bag hours of data found a correlation between measured volume and predictive value, supporting its use in this population.
\end{abstract}

Keywords: Ostomy; remote monitoring; stoma bag; machine learning; thermistor; capacitive sensor.

\section{Introduction}

Abdominal surgery, which may be done in emergency or elective situations, frequently results in the formation of an intestinal stoma. Most commonly fashioned as ileostomy or colostomy, stomas are artificial openings of the bowel onto the surface of the abdomen and are formed when the usual anatomy has been disrupted or in order to protect distal structures from the flow of feces. Conditions for which stoma may result includes inflammatory bowel disease (ulcerative colitis and Crohn's disease), colorectal cancer and the complications of diverticular disease [1]. Population prevalence rates as high as 2-4 per thousand have been reported [2], with 
approximately 100,000 patients with a permanent stoma in the UK, 750,000 in the USA and $1,000,000$ in China [3].

Complication rates for stoma patients exceed 55\% [4] and in a review of all US healthcare data, the all cause 30-day readmission rate for ileostomy patients matched kidney transplant at $29.1 \%$ [5]. Commonly reported complications include acute kidney injury, skin complications and a reduced quality of life [6]. Dehydration is the most common cause for readmission present in approximately $40 \%$ and the subsequent impact on the kidneys can lead to renal failure which is consistently reported in $17-26 \%$ of patients [5,7]. Dehydration and acute kidney injury may be precipitated by high output stoma (HOS), defined as ostomy output $>1500 \mathrm{ml} / \mathrm{d}$ for two or more consecutive days. HOS occurs in up to $17 \%$ of stoma patients and results from bowel wall edema and motility changes that follow surgery [6]. Up to $71 \%$ of patients who develop HOS develop acute kidney injury. Trace element deficiencies (magnesium, phosphate and calcium) are also common in this group. Patients that develop postoperative HOS stay in hospital longer (18 vs 12 days in one study) [6].

Leakage from over-full appliances results in skin complications, which can range from mild cutaneous inflammation to significant ulceration and are observed in $18-55 \%$ of stoma patients [8]. Peristomal skin problems account for more than 1 in 3 visits to ostomy nurses and early intervention can prevent complications and improve outcomes for the patient [9].

The cumulative impact of the complications listed above also imparts a significant burden on the quality of life (QOL) of stoma patients [3]. A systematic review assessed the results of 14 studies investigating quality of life in colorectal cancer stoma patients. Despite variation in methodology, all studies demonstrated that living with a stoma influenced the overall QOL negatively. Painful or irritated peristomal skin, odor, and noise from the appliance were the most commonly reported ostomy-related difficulties [10].

Although some tools exist, there remains a lack of validated and reliable measures of peristomal complications [9]. Equally, efforts to improve outcomes in ostomy patients have focused on education and self-management, often involving manual measurement of stoma output which is unacceptable to many [11]. As a result, patients and healthcare professionals struggle to measure stoma output and monitor skin condition accurately.

Advances in sensor technology and mobile connectivity raise the prospect of continual background monitoring of stoma function. Previously, we designed, fabricated and tested a wireless, flexible and adherent sensor with Bluetooth connectivity capabilities for measuring volume output in ostomy patients [12]. The volume sensor is a flexible polymer strip embedded with conductive materials. When a known voltage is applied to the strip, the resistance can be measured. As the strip is flexed, the conducting particles become further apart, increasing the 
resistance of the circuit through a piezoelectric effect. We were able to demonstrate that a flexible external sensor, affixed to the outside of a stoma bag and wirelessly connected to a patient's smartphone can accurately and reproducibly measure stoma output [13]. The device was also found to be acceptable and usable to patients with newly formed ileostomy [14].

A limitation of the flexible sensor approach is that it requires the volume of the patient's stoma bag to be known. In addition, this method is susceptible to noise and cases were seen where it markedly affected the accuracy of the output recording. Noise was greatest during physical activities and during bag placement. Despite these limitations, it was estimated that such a device could provide a cost saving against current treatment methods up to 33\% [15].

In this work we propose and develop a novel sensor platform for the non-invasive monitoring of stoma output in real time. The sensing components, monitoring temperature change and the presence of liquid, are integrated into an otherwise normal stoma bag. Information on the volumetric filling of the bag is relayed to the patient via a Bluetooth connection to a smartphone, and displayed in an installed application to the patient and clinical team.

\section{Materials and Methods}

\subsection{Characterization of Negative Temperature Coefficient Thermistors}

Thermistors are a kind of semiconductor for which the resistance changes with temperature. The resistance of some types of thermistors decrease with the increase in temperature and are known as negative temperature coefficient (NTC) thermistors. Properties of high resistivity allow for the design of a small mass unit with a fast response, and high sensitivity of the temperature measuring element allows for accurate temperature measurement in a narrow temperature range, suitable for biomedical applications [15].

Temperature is derived from resistance using the Steinhart and Hart Equation where $\mathrm{A}_{0}, \mathrm{~A}_{1}, \mathrm{~A}_{3}$ are constants [16].

$$
1 / \mathrm{T}=\mathrm{A}_{\mathrm{o}}+\mathrm{A}_{1} \ell \mathrm{nR}_{\mathrm{T}}+\mathrm{A}_{3}\left(\ell \mathrm{nR} \mathrm{R}_{\mathrm{T}}\right)^{3}
$$

The relationship between resistance and temperature is linear. The resistance tolerance is reported as $+/-1 \%$. The typical size of an NTC thermistor for an industrial or biomedical application is $1.0 \times 0.5 \mathrm{~mm}$ [17].

\subsection{Characterization of Capacitive Sensors}

Capacitive sensors are used in multiple industrial and consumer applications. Here we describe the use of electrode pads etched from copper and plated with gold for capacitance detection, an 
108

109

110

\section{1}

112

example of which is shown in Figure 1. The copper pads are connected to a capacitive sensing controller. The controller generates the capacitive value for each electrode in the circuit. Capacitance depends on the dielectric constant, the area of the conducting plate, and the separation distance of the plates. If the properties of a capacitive sensor remain fixed, except for its dielectric constant, then the capacitance in terms of its dielectric constant can be calculated as follows:

$$
C\left(\varepsilon_{r}\right)=\varepsilon_{r}\left(\frac{\varepsilon_{0} A}{d}\right)
$$

Where $\mathrm{C}$ is the capacitance in farads $(\mathrm{F}), \varepsilon_{\mathrm{r}}$ is the relative static permittivity (dielectric constant) of the material between the plates, $\varepsilon_{0}$ is the permittivity of free space, $A$ is the area of each plate in square meters, and $\mathrm{d}$ is the separation distance of the two plates [18].

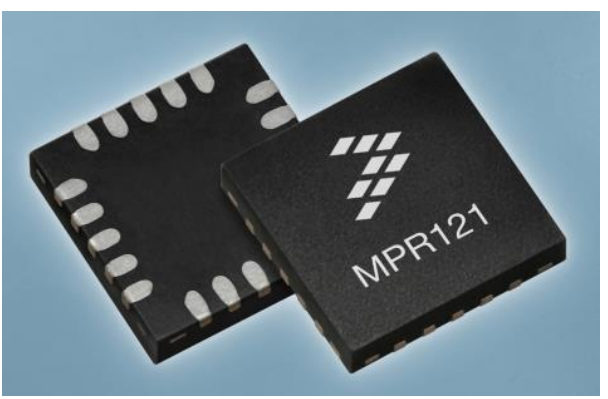

Figure 1. Top and base view of a 20-pin capacitive sensor. The dimensions are $3 \times 3 \times 0.65 \mathrm{~mm}$ [18].

If mounted on the external layer of a plastic fluid receptacle, the wall of the receptacle becomes the dielectric material. When the receptacle is empty the capacitance will be high. When fluid comes into contact with the layer directly on top of the electrode it forms a simple parallel plate capacitor. In this condition the capacitance will drop providing a measurable event that can indicate the presence or absence of fluid. This is demonstrated in Figure 2.

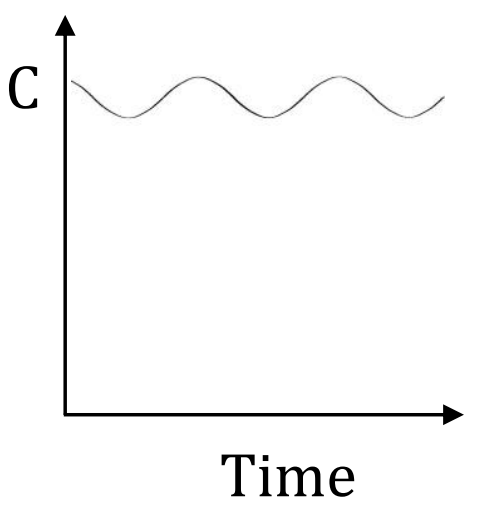

\section{Time}



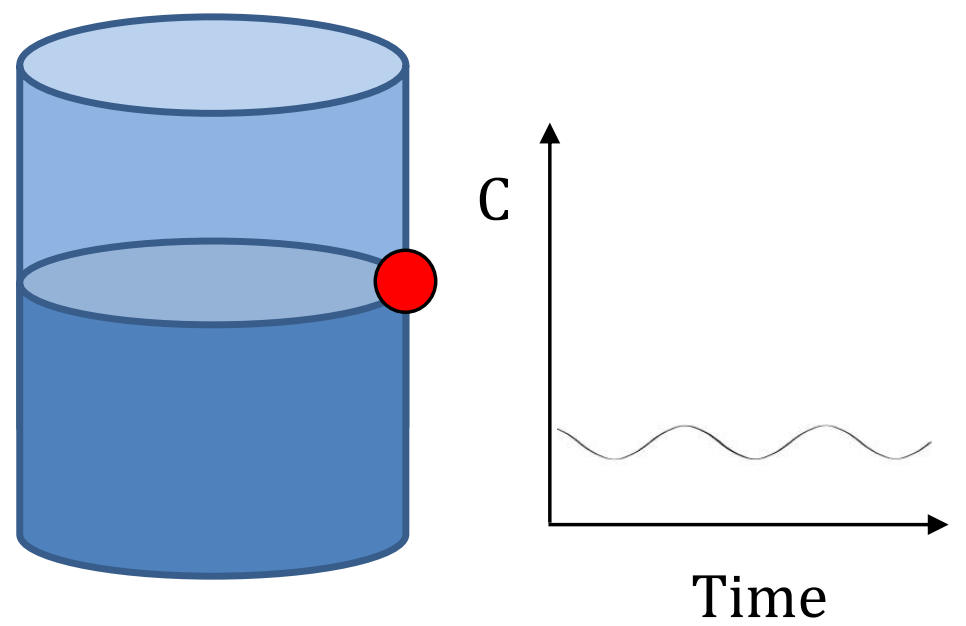

139 B

141 Figure 2. The effect of the presence of fluid on a capacitive sensor (red dot) mounted on the outside 142 of a plastic-walled receptical the capacitance (C) is shown on the y-axis of the graph above and can 143 be seen to be elevated when the sensor is uncovered (A) and drop when the capacitive sensor is in 144 contact with fluid (B).

\subsection{Rationale of using thermal and capacitive monitoring to monitor human stoma ouput}

In health, core body temperature is tightly maintained by physiological mechanisms in a range of $35.5-37.5{ }^{\circ} \mathrm{C}$ and the intestinal lumen environment is maintained at core temperature [19]. Feces therefore exits the body at core temperature. In the case of a patient with an intestinal stoma, output is contained by an appliance, usually a stoma bag. Stoma output occurs in discrete episodes and passes into a stoma appliance in a pattern dictated by its water content, existing effluent within the

151 bag, and distortion of the usual bag shape.

152 As an initial proof of concept, simulated stoma output (oatmeal and apple sauce) was infused

153 into a regular stoma bag and optical and thermal images taken of the process (FLIR E60 thermal

154 imaging camera). This confirmed the hypothesis that as effluent settles within the bag, a thermal

155 boundary will become apparent that demarcates the accumulated level of fecal output. This is 156 demonstrated in Figure 3. 

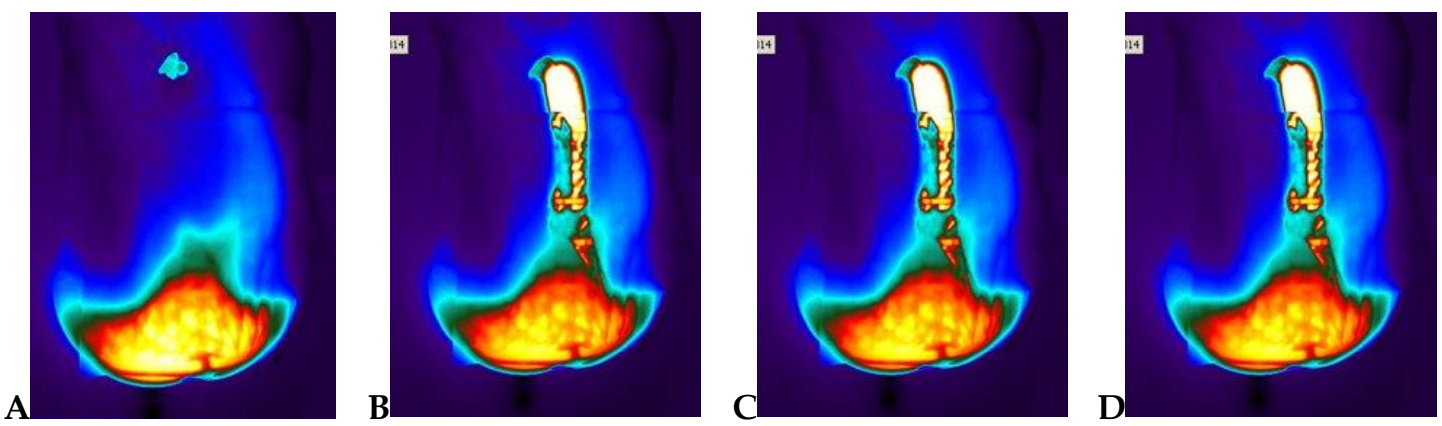

159 Figure 3. Appearance of a thermal boundary within a stoma bag during infusions of simulated feces.

160 The boundary was still visible 5 minutes after the infusion finished; the path that the effluent flowed

161 through, remained heated 5 minutes after infusion finished. Pictures from left to right are: (A) before

162 infusion, (B) during infusion, (C) right after infusion finished, and (D) 5 minutes after infusion

163 finished. Note that bright yellow indicates the highest temperature and dark blue indicates the lowest

164 temperature in these infrared images.

\section{2.4. Development of a volumetric array: proof of concept}

166 The validity of using a thermistor array, externally mounted, to determine dynamic episodes of

167 stoma output and bag filling level was investigated. An array of NTC thermistors mounted on a

168 flexible polymer (Murata Manufacturing, Kyoto, Japan) was adhered to the outer layer of a standard

169 ostomy bag $450 \mathrm{ml}$ 2-piece drainable unit, Coloplast, Humlebaek, Denmark). The full set up is

170 demonstrated in Figure 4 below. 


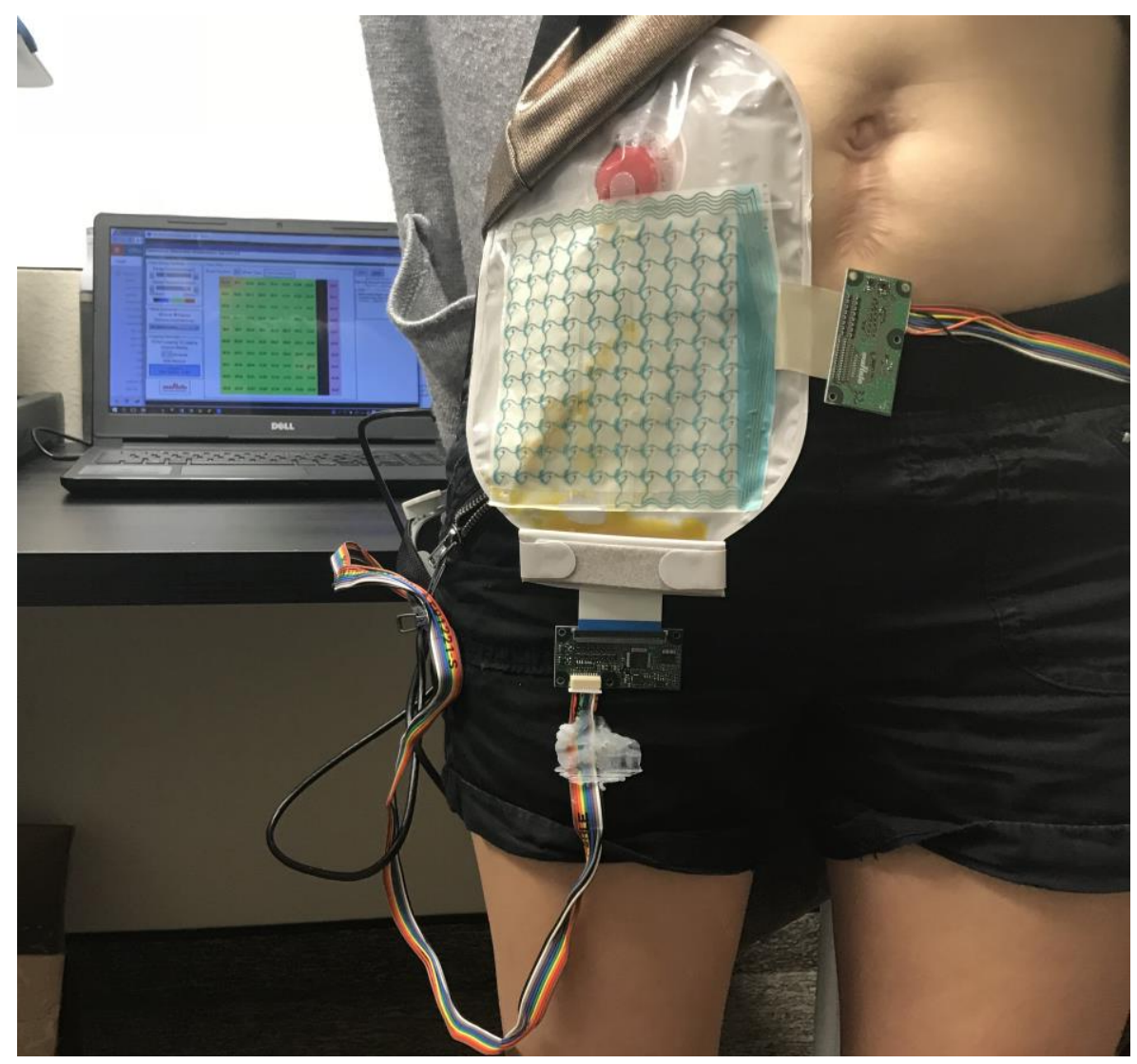

173 Figure 4: Experimental set up to investigate the performance of a thermistor array as a method of

174 detecting the function and cumulative output of an excretory stoma.

175

Simulated stoma effluent (Motts Apple Source, Motts LLP, Plano, Texas) was added in 50ml

177 aliquots, previously heated to body temperature $\left(37^{\circ} \mathrm{C}\right)$ whilst temperature change within the bag

178 was monitored in real time. As can be seen in Figure 5, the pattern of temperature change was distinct

179 at different temperature levels at a single thermistor. In addition, the flow-path of the infusion and

180 thermal barrier of effluent settlement was visible across the entire thermistor array in a temperature

181 visualization during sequential simulated outputs. This is shown in Figure 6 below. 


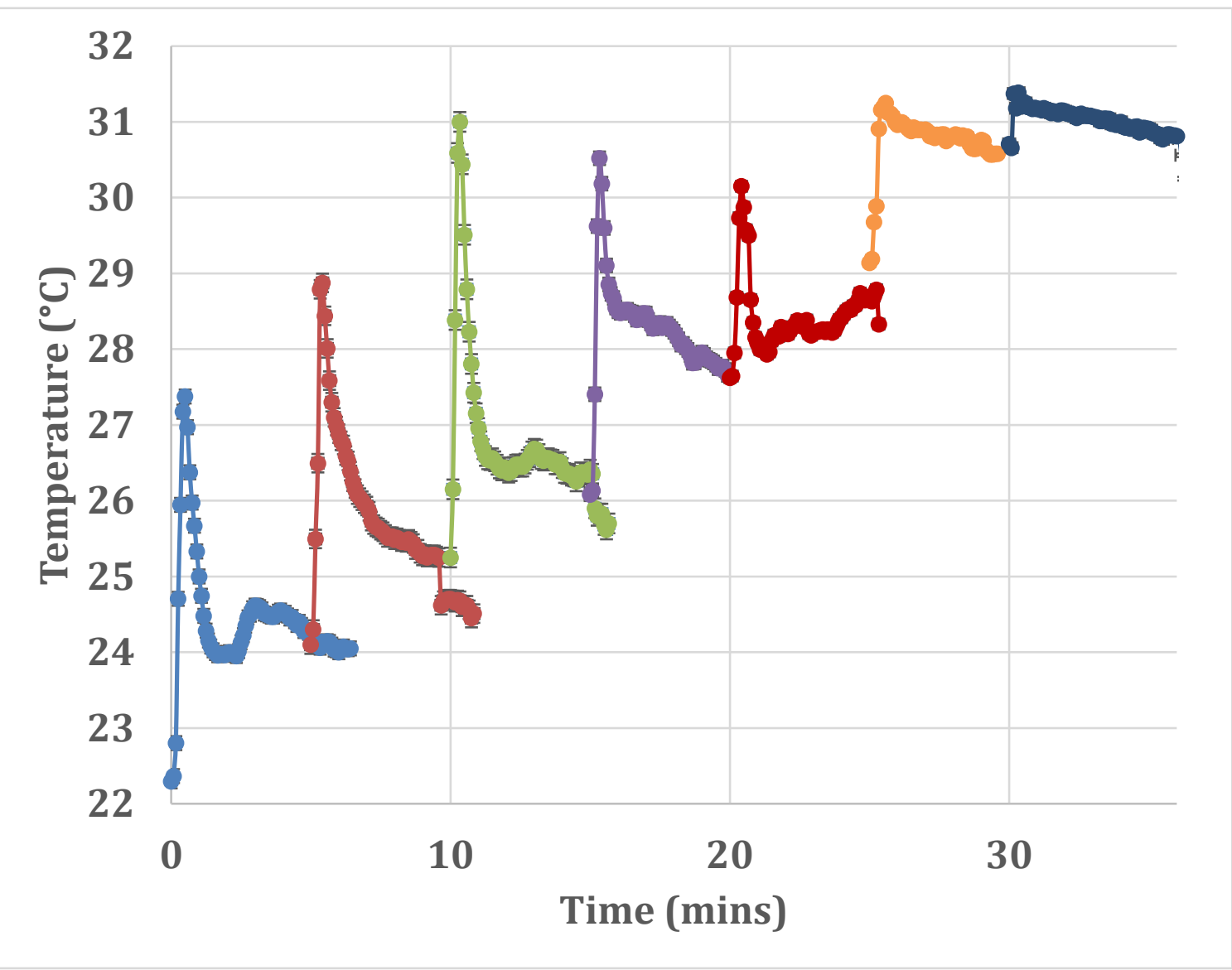

182

Figure 5: Average temperature at a single thermistor for volumes of simulated effluent infused at

184 body temperature into a prototype 'smart' stoma bag in the up-right/ supine position. Standard error 185 also plotted. Each color represents an additional infusion in $50 \mathrm{ml}$ aliquots up to a total of $350 \mathrm{ml}$.

186
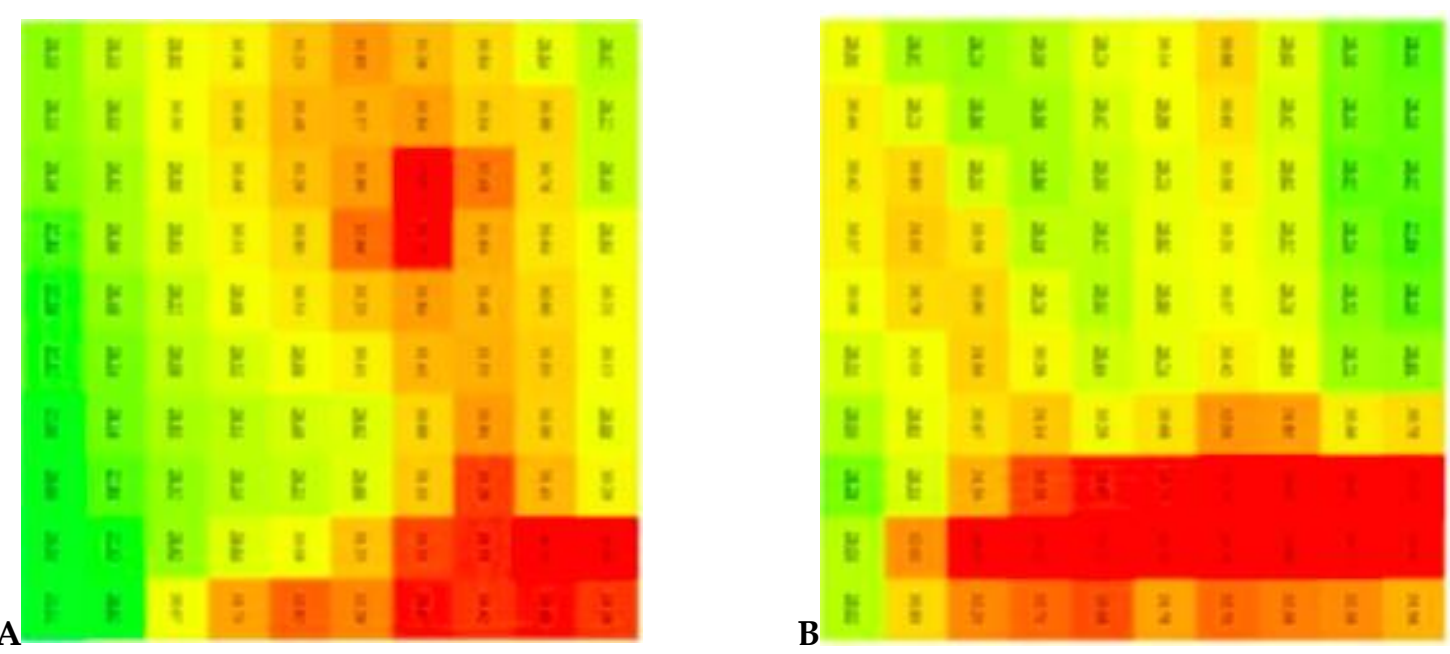

188 Figure 6: 'heat-map' representation of the thermistor array used in the experimental set-up 189 demonstrating the detection of the flow-path of an infusion of simulated effluent (A) and thermal 
190

191 physical orientation during the experiment.

\subsection{Development of a capacitive array: proof of concept}

The validity of using capacitive sensor technology, externally mounted, to determine the amount of volume filled in the bag was tested in early 2018. A Cypress CapSense Liquid Level sensor (20) was mounted on the outer layer of a standard ostomy bag 450ml 2-piece drainable unit (Coloplast, Humlebaek, Denmark) as seen in Figure 7. The bag was worn by a non-ostomate volunteer.
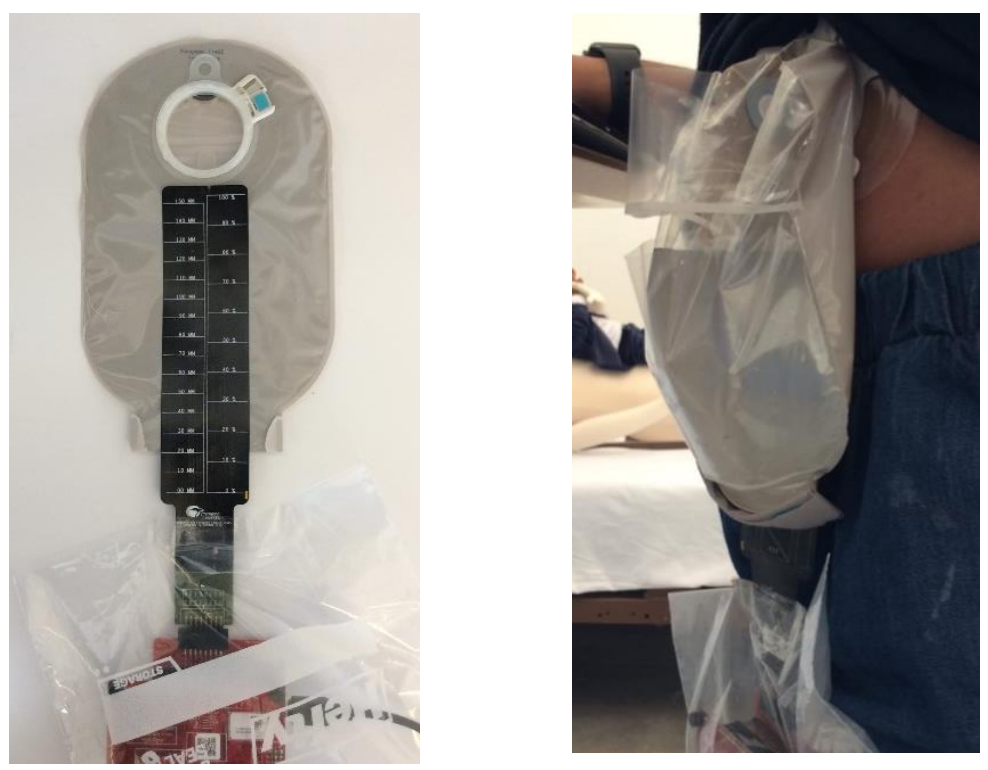

Figure 7: A Cypress CapSense Liquid Level sensor mounted on the outer layer of a standard ostomy bag 450ml 2-piece drainable unit (Coloplast, Humlebaek, Denmark). The bag was worn by a nonostomate volunteer.

The sensing device used deploys 12 capacitive sensors placed in a vertical strip pattern. The 211 sensor has been designed such that the absolute value of each capacitive sensor is not important for 212 determining whether the liquid is present or absent. The sensor pattern is segmented allowing each 213 sensor element to accurately measure the liquid level in its limited range. The reported liquid height 214 level is the highest sensor covered with liquid.

215 The mounting on the bag was done via a pre-applied 3M adhesive on the capacitive sensor flex board, which allowed this board to be directly adhered to the outer layer of the bag. 
Fluid was added in $50 \mathrm{~mL}$ aliquots (to a total of $350 \mathrm{~mL}$ ). The capacitive flex PCB used, was 218 connected to a Liquid Level Sensing Arduino Shield board and the signal of the sensor, as a function 219 of the infusion was monitored on opensource software. The output of the sensor was in terms of the 220 height of the liquid being measured in $\mathrm{mm}$ as a function of time - datapoints were collected every 221 second.

Figure 8 shows a graph of how the capacitive sensor board responded to an incremental increase of volume in the bag in segments of $50 \mathrm{~mL}$. The data shows a stepwise increase as a function of increasing volume in the bag. The step wise increase of the sensors is a result of the binary output of each sensor, resulting in the volume increase to look like an increasing step.

The graph shows that for each infusion, the height fluctuates but eventually plateaus at the corresponding increased height. The noise/fluctuations seen in the data may be as result of the movement of the volunteer, although their basic position was to stand throughout the duration of the test.

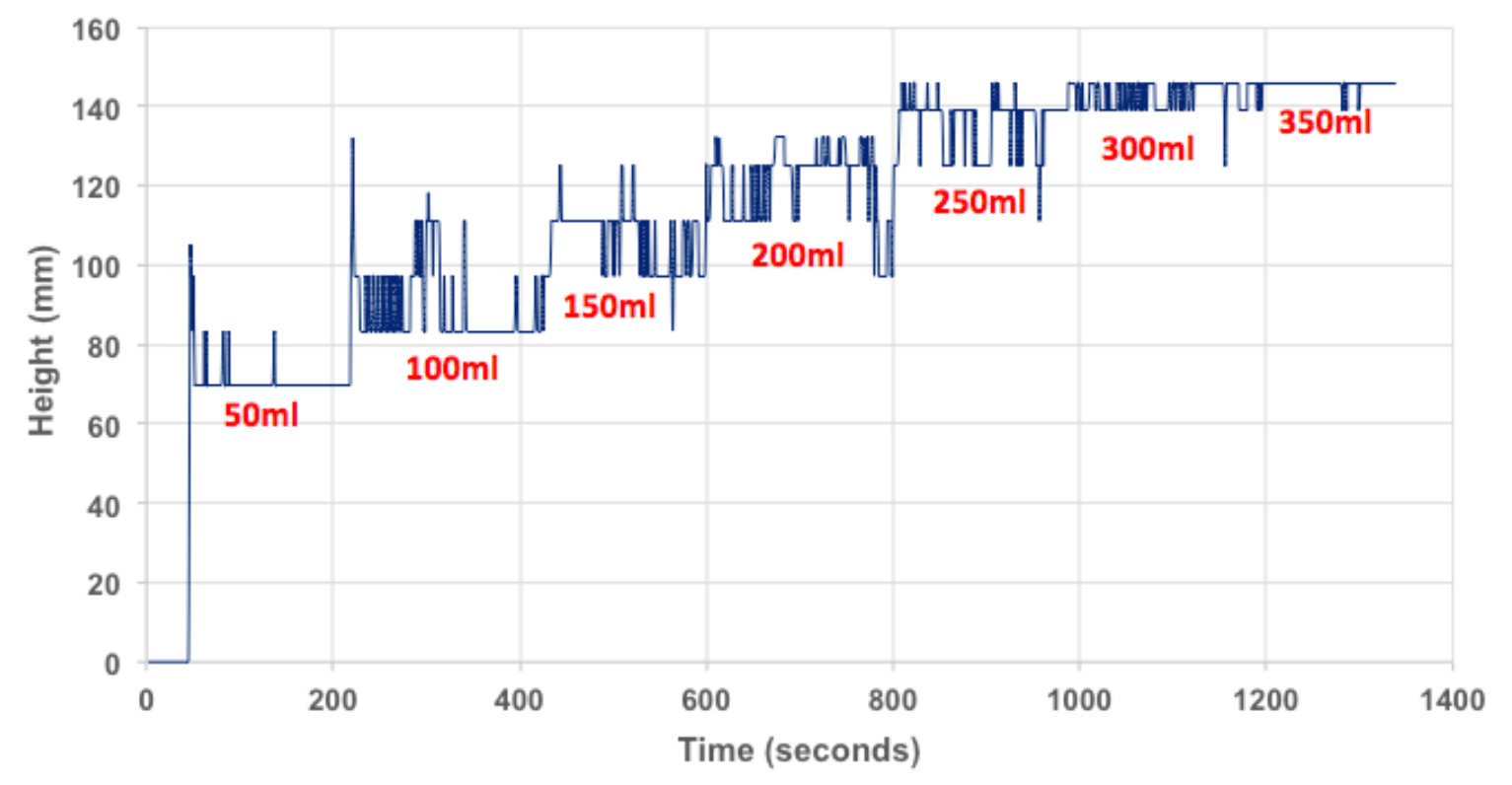

232 Figure 8: Graph showing the response of the Cypress CapSense Liquid Level Sensing Sensor, in the 233 form of height, as a function of time, during which there was an incremental increase in volume of 234 the ostomy bag, in gaps of $50 \mathrm{~mL}$, up to $350 \mathrm{~mL}$ (indicated in red on the graph). 


\subsection{Integration of a volumetric sheet into a standard stoma bag}

238 On the basis of this data, an array of thermistors and capacitive sensors was built into a flexible

239 polyamide substrate. This was incorporated into the structure of a stoma bag and detect the thermal

240 and capacitive changes present during stoma output. The board is equipped with a coin-cell battery

241 for power supply and an NFC chip for data transfer. The integrated 'smart' stoma bag is shown in 242 Figures 9 and 10 below.

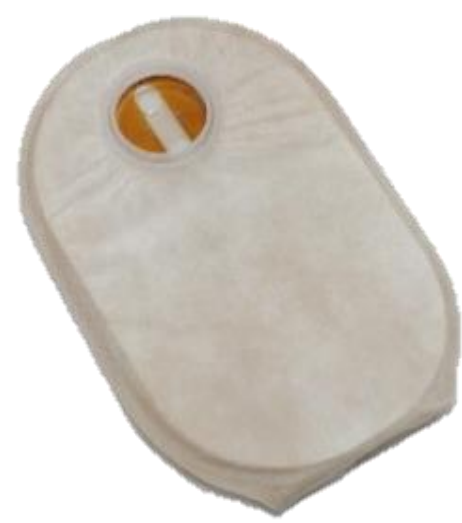

243 A

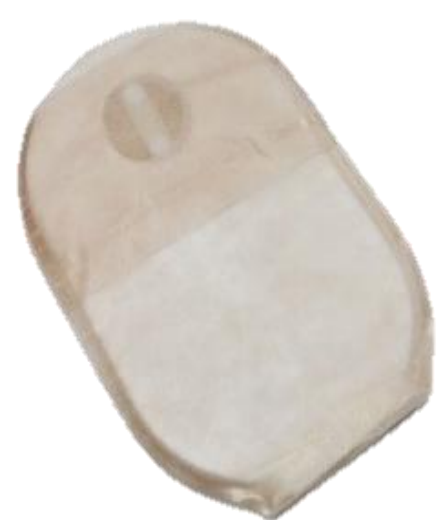

B

244 Figure 9: Front (A) and back (B) images of a 'smart' stoma bag integrated with a volumetric sensor 245 array and wireless connectivity capability.

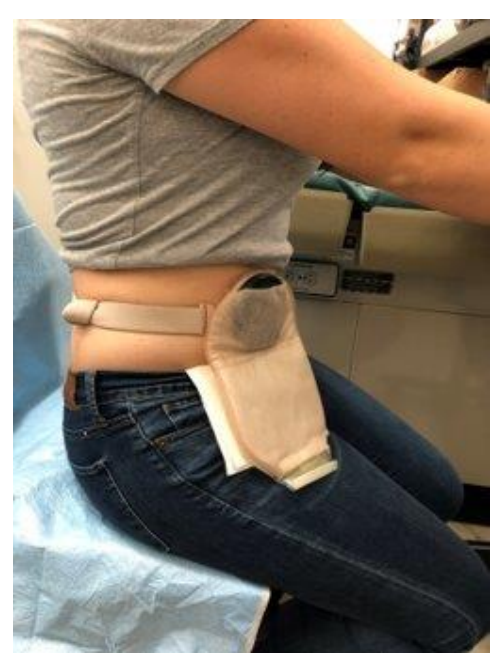

248 Figure 10: A 450ml ostomy bag containing an integrated volumetric and capacitive board. In this case

249 a fully wireless 'hub' device (black circular device in the front pocket of the stoma appliance) was 250 used to connect with the volumetric array by NFC and to an installed smartphone application by 251 Bluetooth low energy (BLE). 


\subsection{Communication Protocol}

The sensing components communicate with themselves via NFC. A BLE chip transmits data to a smartphone application. Data is then transmitted via HTTPS protocol to a secure cloud server. In all cases, the data transfer is HIPAA (The Health Insurance Portability and Accountability Act) compliant and encrypted thus preventing the data from being hacked or compromised by any 3rd party.

\subsection{Software Applications}

A companion smartphone software application was developed to support use of the integrated sensing device. This incorporates administrative tools, data visualization and access to educational and care resources. Alerts can be set allowing the patient, or the clinicians who are monitoring the patient, to take preventative action to avoid complications.

\subsection{Cloud Architecture}

The cloud architecture is designed so that data from the sensors can be sent in real time to a patient's or clinician's mobile device. All these data can be accessed through a dedicated HIPAA compliant cloud service. The cloud service and communication system are secured in five layers: all patient data is TLS (Transport Layer Security) secured in transit between smart bag and the cloud and between cloud and software apps; patient personal data in cloud is encrypted at rest; 3rd party cloud service provides its own data protection; all patient data is encrypted again when requested from the cloud and only authorized internal employees and registered users can view the data; internal electronic devices storing trial data are password protected and all data is backed up to a HIPAA compliant cloud library. The communication flow is demonstrated in Figure 11.

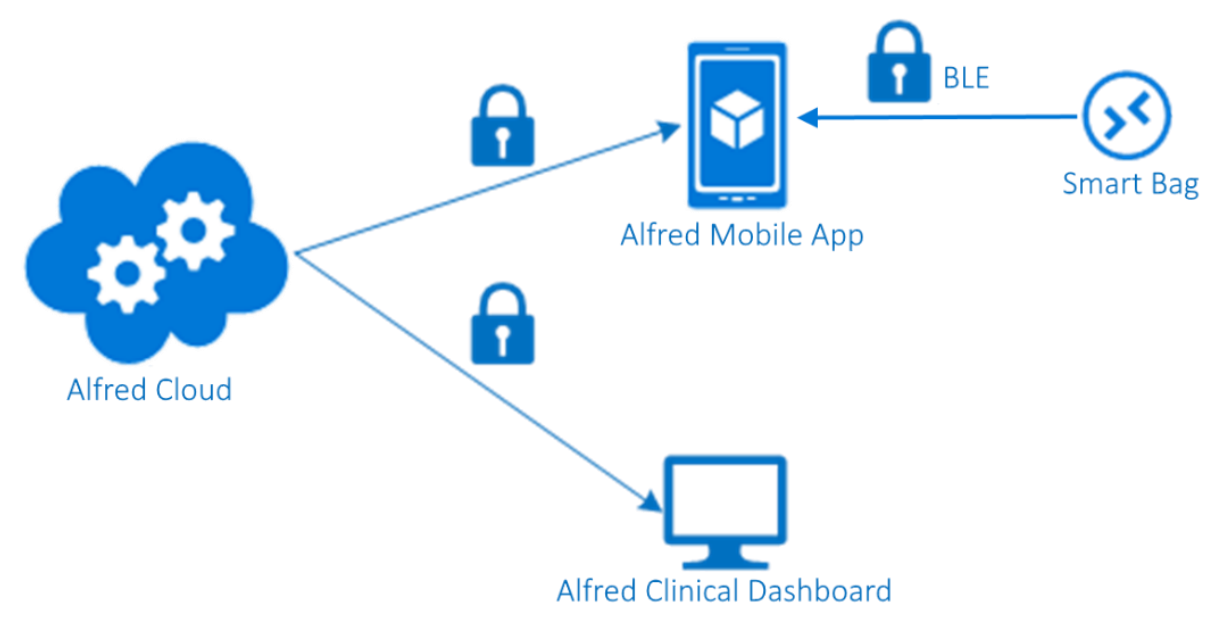

276 Figure 11. Overview Diagram of data communication flow chart of the connected ostomy bag 


\section{Data extraction from volumetric sensor array}

\subsection{Design of a machine learning algorithm}

Direct reading of fluid level by thermistors or capacitive sensors can be inaccurate in dynamic environments. Experience from the use of capacitance for fluid level sensing in industry has also demonstrated the issue of error due to adherence of material, presence of contaminants or movement of the storage vessel. Several methods have been used to compensate for these dynamic effects and artificial neural networks (ANN) have been found to provide an effective method to allow capacitive sensors to function in a dynamic environment without compromising accuracy [21].

We therefore developed a machine learning algorithm to account for the type of output and calculate a reliable volume. For the purposes of processing this data, we selected a static feedforward neural network to account for both the data from thermistors and capacitive sensors. Resilient backpropagation algorithm (RPROP) was used for supervised training of the multilayer perceptron.

Feed-forward Backpropagation (BP) neural network is a static network, where signals travel in one direction only, i.e. from input to output. There is no loop or feed-back between neurons and their inputs and outputs. Backpropagation network topology is extensively used in pattern recognition. Figure 12 illustrates the structural diagram of the BP network that takes the signal features and produces the fluid volume as the output.

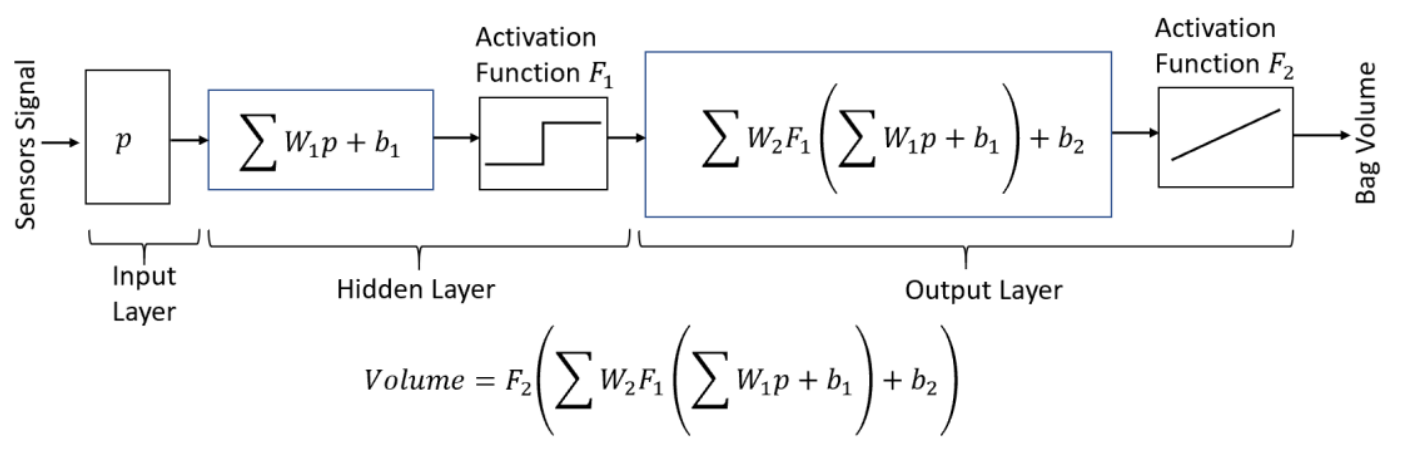

Figure 12. Structural diagram of the BP network that takes the signal features and produces the fluid volume as the output.

\subsection{Training of a machine learning algorithm}

In order to predict volume based on sensors readings, data was acquired corresponding to each vector. Simulated effluent (water and apple sauce) was infused in $50 \mathrm{ml}$ aliquots multiple times in a bag of set volume, producing the volume range from 50 to $350 \mathrm{ml}$. Multiple cycles resulted in a training set size of about 1500 data points representing 1500 values of volume. 
305

306

307

308

309

310

311

312

313

314

315

316

317

318

319

\section{Multilayer Perceptron Architecture}

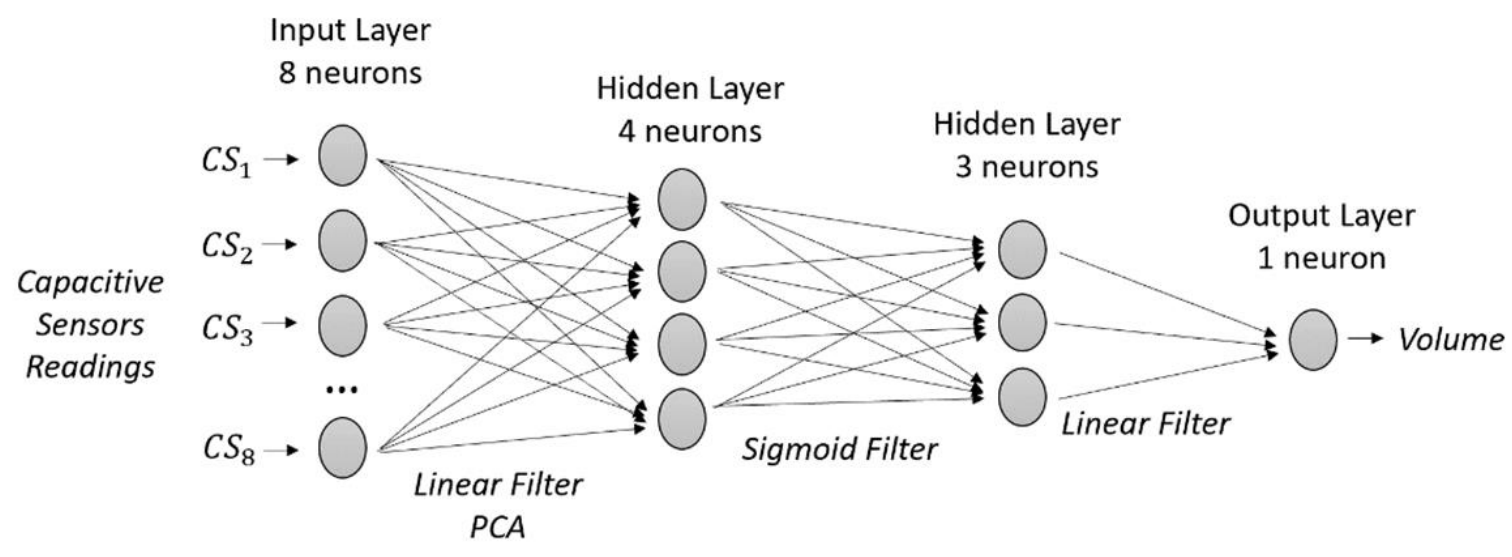

Figure 13. The multilayer perceptron architecture designed for volumetric prediction based on sensors signal.

The model was trained on $80 \%$ of the dataset. The cost function for that training is shown on Figure 14. We see that the solution converges within several hundred iterations and the mean square error was reduced to $32.6 \mathrm{ml}$. The model was validated on the remaining $20 \%$ of the dataset with Mean Square Error equal to $35.2 \mathrm{ml}$. This is equivalent to $10 \%$ of the $350 \mathrm{ml}$ maximum effluent volume infused.

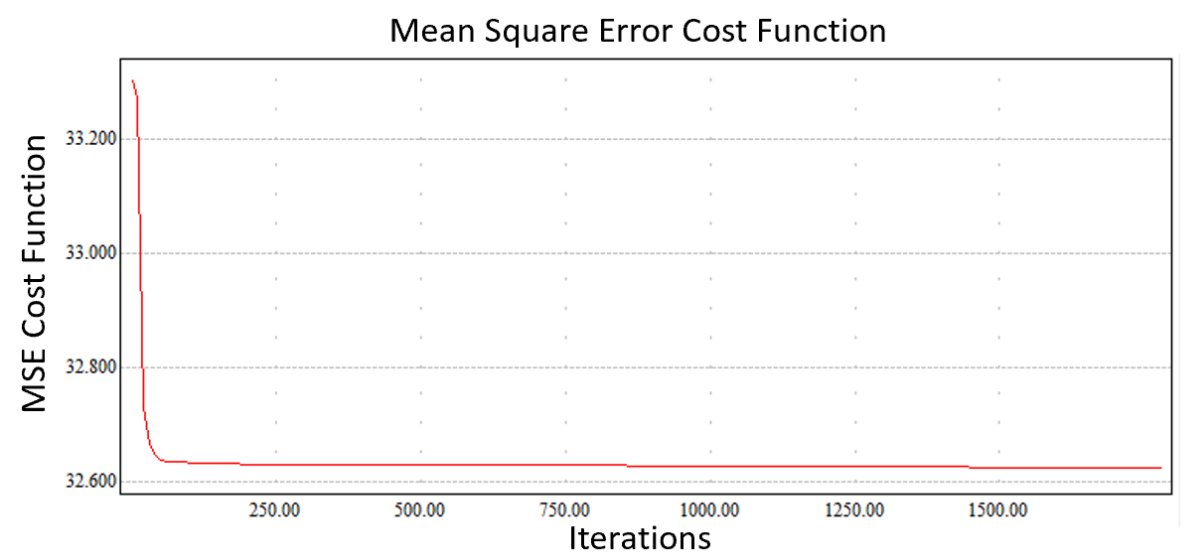

This dataset was used to train the model. Below is the schema for multilayer perceptron architecture designed for that task. It has one input layer and 2 hidden layers of reducing size. The output layer is a single neuron (which is the output volume). Figure 13 illustrates the multilayer perceptron architecture designed for volumetric prediction based on sensor signal. 
321 Figure 14. Mean square error cost function for a training set of simulated effluent infusions into a 322 'smart' stoma bag at volumes between 50 and $350 \mathrm{ml}$.

323 5. Results

\section{5.1. Non Ostomate Testing}

325 The prototype combined system integrated into a $450 \mathrm{ml}$ two-piece drainable stoma bag was 326 tested in a simulated real-world setting. Two volunteer non-ostomates wore the stoma bag system in 327 a standing, sitting or supine position. Simulated effluent pre-heated to body temperature $\left(37^{\circ} \mathrm{C}\right)$ was 328 added in $50 \mathrm{ml}$ aliquots every 3 minutes until $350 \mathrm{ml}$ had been instilled. The bag was then drained and 329 the infusion process repeated.

330 Filling of simulated effluent infused at body temperature, was detectable by temperature increase 331 in all thermistors, greatest in the region of settled fluid. The baseline temperatures were increased 332 compared to bench-testing due to the contribution of body heat. The temperature differentials 333 between filling events was smaller in sitting and supine positions. Capacitance and temperature drop 334 in the lower bag region was found to be an accurate method of detecting bag drainage.

\section{5.2. Testing on Existing Ileostomy Patients}

336 Eleven existing ileostomy patients (7F, median age 33) were recruited to a single center. Data 337 was collected prospectively, and the device performance was described. Participants wore the fully 338 integrated and wirelessly connected smart stoma bag for up to 7.5 hours (median 3.0 hours) and all 339 stoma output was recorded at the point of bag drainage. To mimic real-word conditions, participants 340 were permitted to eat and drink ad-libitum, and could drain their stoma bag when $50 \%$ full or greater. 341 Although exhaustive exercise was prohibited, there were no restrictions on position, movement or 342 physical activity. The primary outcome was validation of the algorithm to calculate stoma output 343 volume from thermal and capacitive measurement within the SmartBag system.

344 A total of 51.6 bag hours of data were collected. In this time $3139 \mathrm{ml}$ of stoma output was 345 measured. Mean (standard deviation) stoma output across all participants was $53.3(42.7) \mathrm{ml} / \mathrm{h}$. The 346 algorithm predicted output of $3332 \mathrm{ml}$ in the same time period, providing a predicted mean stoma 347 output per participant of 56.3 (45.5) $\mathrm{ml} / \mathrm{h}$. Figure 15 below demonstrates the relationship between 348 measured and predicted stoma output volumes in real ostomy patients. 


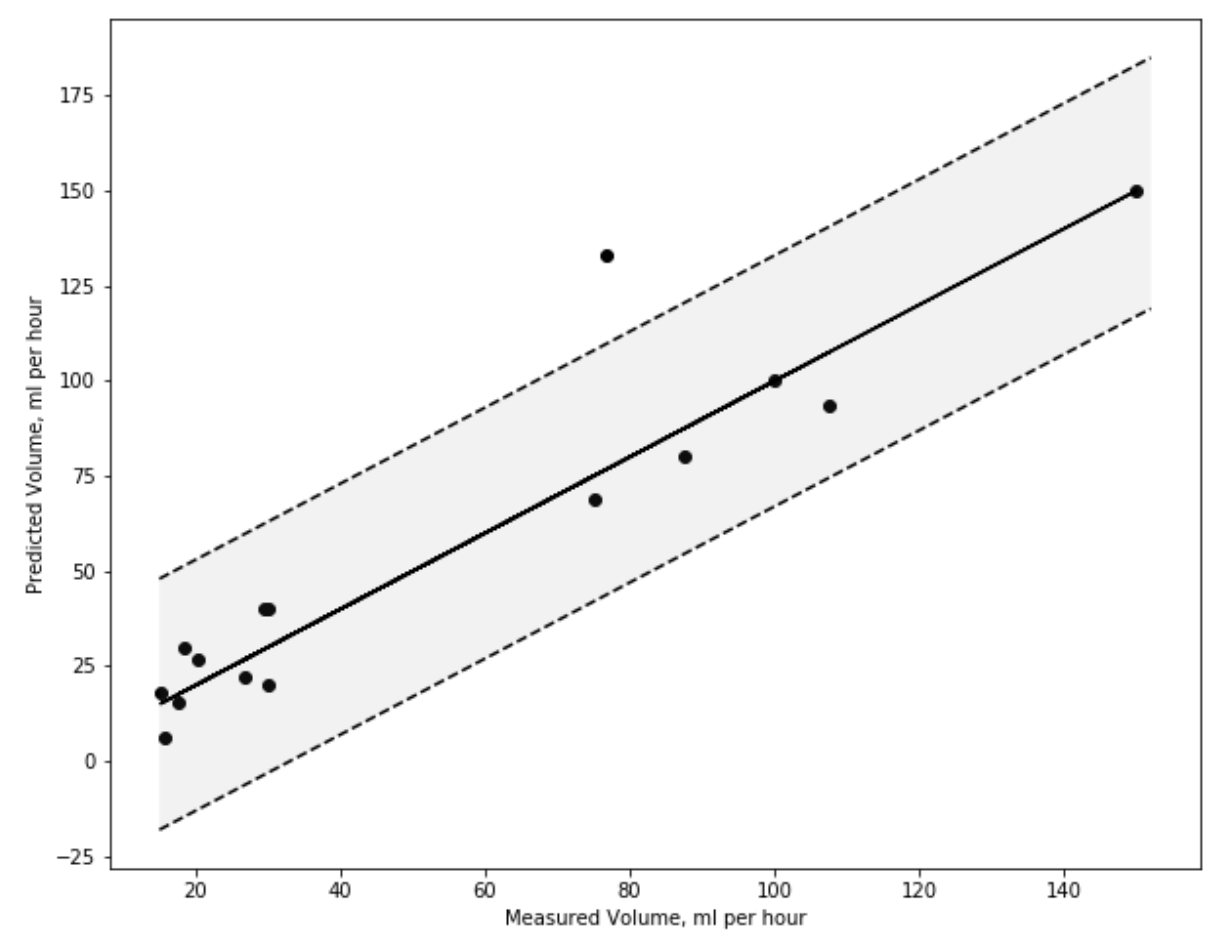

350 Figure 15. relationship between predicted and measured ileostomy output over 16 bag drain events

351 in 11 ileostomy patients.

\section{Discussion}

Here we have demonstrated that a volumetric array consisting of thermistors and capacitive sensors can be mounted on a flexible polyimide substrate measuring $18 \mathrm{~cm} \times 12 \mathrm{~cm}$ and can be completely incorporated into an ostomy appliance measuring $23 \mathrm{~cm} \times 15 \mathrm{~cm}$. The size and appearance of the device is comparable to currently marketed stoma appliances, as can be seen in Figure 9 above.

The theoretical concept of using thermal and capacitive measurement to determine the characteristics of stoma output and cumulative volume have been described. Data is also shown for

360 how the sensors were integrated into a flexible substrate, and how this sensing model can be used to 361 detect volume in a lab environment through the employment of two separate resilient 362 backpropagation algorithms. Finally, the validity of using these methods to detect ileostomy output 363 in a real-world setting on human volunteer ostomates was shown. Whilst artificial neural networks 364 have been used in industrial settings to improve the accuracy of fluid level sensing in dynamic 365 environments, previous solutions were designed to account for 'slosh' caused by variable 366 acceleration in one or two axes of movement. They were also designed to be mounted on the side- 
wall of a rigid structure [21]. A combination of thermal and capacitive sensing has not previously

368 been described in volumetric sensing applications. Furthermore, no prior approach to sensor deployment has ever been shown to be consistently accurate when mounted to a flexible receptacle such as a stoma appliance.

We previously reported the use of flexible piezoelectric sensors that have been attached to the outside of a stoma bag. As the strip is flexed, the conducting particles become further apart, increasing the resistance of the circuit. The device in-situ can be seen in Figure 16. We were able to demonstrate that the changes in resistance upon flexing, correlated to the filling status of a stoma bag when its capacity is known. In simulated real-world conditions the volumetric data was found to be consistent and accurate in a series of ostomates who used the device. This device was found to be acceptable and usable, but did not provide a consistent accurate reading due to connectivity issues and a high noise to signal ratio in a dynamic environment [14].

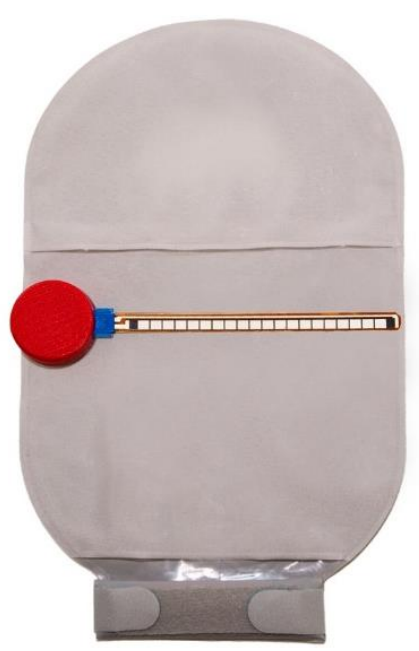

Figure 16 : Example of a flexible piezoelectric sensor in situ attached to a stoma bag.

The solution described here, overcomes these problems by employing two sensing modalities and an artificial neural network to determine accurate volume of bag content in real time, whilst adding to a cumulative total only when a drain is detected.

The ileostomy population suffers disproportionate morbidity, particularly with regards to dehydration and acute kidney injury [6]. Such complications are frequently associated with high output stoma which also contributes to macro and micronutrient losses [22]. The impacts of such complications are significant, with negative effects on quality of life frequently reported in this group [10], particularly in the domains of physical, social, psychological, and spiritual well-being [23]. The cost to the healthcare economy is also great. In an economic analysis, the direct hospital costs of patients with a stoma was over twice as high as similar patients who do not receive a stoma $(\$ 26,917$ vs. $\$ 13,817$ [24]. Other studies have also consistently found that acute healthcare resource utilization 
393 is higher than expected in stoma patients [25]. Recent studies have advocated better monitoring of

394 ostomates and tailored interventions to reduce complications and improve self-monitoring $[5,26]$.

395 This does not only apply to the outpatient setting. Length of stay is significantly longer in patients

396 that develop high stoma output and this is at least in part due to the need of the clinical team to have

397 confidence in the accurate monitoring of output volumes [6]. Inpatient documentation of fluid

398 balance is known to be inaccurate and creates a considerable burden for clinical staff to document,

399 not to mention risks involved in handling potentially hazardous bodily waste for extended periods

400 [27]. It is reasonable that a remote monitoring system that can securely communicate instantaneous

401 and cumulative output data to the patient and their clinical team, could contribute to improved 402 outcomes in this population.

\section{Patents}

404 The discussion of the foregoing technology is covered by U.S. Patent Application No. 16/184764, 405 16/184787, and 16/184828, which were published on May 9, 2019, with the United States Patent and 406 Trademark Office.

407 Author Contributions: Conceptualization, R.F. and A.N.; methodology, R.F., Y.W., A.N. and P.B.; software, I.D.; 408 hardware, D.R., validation, I.D., A.N. and R.F.; formal analysis, R.F..; investigation, A.N., D.R., P.B., I.D. and 409 Y.W.; resources, A.N.; data curation, I.D.; writing - original draft preparation, R.F.; writing - review and editing, 410 C.L.; visualization, A.N. and I.D.; supervision, C.L.; project administration, R.F.; funding acquisition, R.F.

411 Funding: This research was funded by the Small Business Research Initiatives grant (grant number A-0208) 412 awarded by SBRI Healthcare, a part of NHS England.

413 Acknowledgments: We thank Dr. Chehkuan Tai for her assistance in compiling, proof-reading and submitting 414 this manuscript.

415 Conflicts of Interest: R.F. is the medical director for 11 Health and Technologies Inc. He is paid a salary to 416 coordinate and oversee clinical research and development activities. I.D, A.N., P.B. and Y.W. are employees of 41711 Health and Technologies Inc. C.L. is a member of the clinical advisory board for 11 Health and Technologies 418 Inc. C.L. is paid an honorarium for this role. The funders had no role in the design of the study; in the collection, 
1. Phatak, U.R.; Li, L.T.; Karanjawala B.; Chang G.J.; Kao L.S. Systematic review of educational interventions for ostomates. Dis Colon Rectum. 2014;57(4):529-37.

2. Thorpe, G.; McArthur, M.; Richardson, B. Healthcare experiences of patients following faecal output stoma-forming surgery: a qualitative exploration. Int J Nurs Stud. 2014;51(3):379-89.

3. Liao, C.; Qin, Y. Factors associated with stoma quality of life among stoma patients. International Journal of Nursing Sciences. 2014;1(2):196-201.

429 4. Kwiatt, M.; Kawata, M. Avoidance and management of stomal complications. Clin 430 Colon Rectal Surg. 2013;26(2):112-21.

431 5. Justiniano, C.F.; Temple L.K.; Swanger A.A.; Xu Z.; Speranza, J.R.; Cellini, C. et al. 432 Readmissions With Dehydration After Ileostomy Creation: Rethinking Risk Factors. Dis 433 Colon Rectum. 2018;61(11):1297-305.

434 6. Arenas Villafranca, J.J.; López-Rodríguez, C.; Abilés, J.; Rivera, R.; Gándara Adán, N.;

435 Utrilla Navarro, P. Protocol for the detection and nutritional management of high-output 436 stomas. Nutr J. 2015;14:45.

437 7. Paquette, I.M.; Solan, P.; Rafferty, J.F.; Ferguson, M.A.; Davis, B.R. Readmission for 438 dehydration or renal failure after ileostomy creation. Dis Colon Rectum. 2013;56(8):974-9.

439 8. Sarkut, P.; Dundar, H.Z.; Tirnova, I.; Ozturk, E.; Yilmazlar, T. Is stoma care effective in 440 terms of morbidity in complicated ileostomies? Int J Gen Med. 2015;8:243-6.

441 9. Martins, L.; Ayello, E.A.; Claessens, I.; Steen Hansen, A.; Hentze Poulsen, L.; Sibbald 442 R.G. et al. The ostomy skin tool: tracking peristomal skin changes. Br J Nurs.

443 2010;19(15):960, 32-4.

444 10. Vonk-Klaassen, S.M.; de Vocht, H.M.; den Ouden, M.E.; Eddes, E.H.; Schuurmans, 445 M.J. Ostomy-related problems and their impact on quality of life of colorectal cancer 446 ostomates: a systematic review. Qual Life Res. 2016;25(1):125-33.

447 11. Nagle, D.; Pare, T.; Keenan, E.; Marcet, K.; Tizio, S.; Poylin, V. Ileostomy pathway 448 virtually eliminates readmissions for dehydration in new ostomates. Dis Colon Rectum. 449 2012;55(12):1266-72.

450 12. Rajagopal, S.; Fearn, R. A novel wireless device can accurately measure output in 451 ostomy patients (poster presentation). European Society of Coloproctology; Berlin, 452 Germany 2017.

453 13. Rajagopal, S.; Fearn, R. A novel wireless device can accurately measure output in 454 ostomy patients (podium presentation). Society of American Gastrointestinal and 455 Endoscopic Surgeons 16th World Congress; Seattle, Washington 2018.

456 14. Fearn, R. Usability and acceptability of a connected medical device to aid self457 management in ileostomy patients. American Society of Colorectal Surgeons Annual 458 Scientific Meeting; Nashville, Tennessee 2018.

459 15. Kontovounisios, C.; Smith, J.; Dawson, P.; Warren, O.; Mills, S.; Von Roon, A. et al. 460 The Ostom-i ${ }^{\mathrm{TM}}$ Alert Sensor: a new device to measure stoma output. Tech Coloproctol. 461 2018;22(9):697-701.

462 16. Chen, C. Evaluation of resistance-temperature calibration equations for

463 NTC thermistors Measurement. 2009;42(7):1103-11. 
464

465

466

467

468

469

470

471

472

473

474

475

476

477

478

479

480

481

482

483

484

485

486

487

488

489

490

491

492

493

494

17. Panasonic NTC Thermal Management Solutions. Available from:

https://industrial.panasonic.com/cdbs/www-data/pdf/AUA0000/AUA0000COL16.pdf. (Last accessed 5/23/19)

18. Inc. FS. Proximity Capacitive Touch Sensor Controller, Document Number: MPR121.

19. Roberge, R.J.; Kim, J.H.; Yorio, P.; Coca, A.; Seo, Y.; Quinn, T. et al. Umbilical temperature correlation with core and skin temperatures at rest, in the heat and during physical activity. Int J Hyperthermia. 2017;33(7):724-32.

20. CY8CKIT-022 CapSense ${ }^{\circledR}$ Liquid Level Sensing Shield [Available from:

https://www.cypress.com/documentation/development-kitsboards/cy8ckit-022-capsenseliquid-level-sensing-shield. (Last accessed 5/23/19)

21. Terzic, E.; Nagarajah, C.; Alamgir, M. Capacitive sensor-based fluid level measurement in a dynamic environment using neural network. Engineering Applications of Artificial Intelligence. 2010;23:614-9.

22. Messaris, E.; Sehgal, R.; Deiling, S.; Koltun, W.A.; Stewart, D.; McKenna, K. et al. Dehydration is the most common indication for readmission after diverting ileostomy creation. Dis Colon Rectum. 2012;55(2):175-80.

23. Sun, V.; Ercolano, E.; McCorkle, R.; Grant, M.; Wendel, C.S.; Tallman, N.J. et al. Ostomy telehealth for cancer survivors: Design of the Ostomy Self-management Training (OSMT) randomized trial. Contemp Clin Trials. 2018;64:167-72.

24. Damle, R.N.; Cherng, N.B.; Flahive, J.M.; Davids, J.S.; Maykel, J.A.; Sturrock, P.R. et al. Clinical and financial impact of hospital readmissions after colorectal resection: predictors, outcomes, and costs. Dis Colon Rectum. 2014;57(12):1421-9.

25. Tyler, J.A.; Fox, J.P.; Dharmarajan, S.; Silviera, M.L.; Hunt, S.R.; Wise, P.E. et al. Acute health care resource utilization for ileostomy patients is higher than expected. Dis Colon Rectum. 2014;57(12):1412-20.

26. Chen, S.Y.; Stem, M.; Cerullo, M,; Canner, J.K.; Gearhart ,S.L.; Safar, B. et al. Predicting the Risk of Readmission From Dehydration After Ileostomy Formation: The Dehydration Readmission After Ileostomy Prediction Score. Dis Colon Rectum. 2018;61(12):1410-7. 27. Vincent, M.; Mahendiran, T.; Improvement of fluid balance monitoring through education and rationalisation. BMJ Qual Improv Rep. 2015;4(1). 